\title{
Model-based predictive traffic control for intelligent vehicles: Dynamic speed limits and dynamic lane allocation*
}

\author{
L.D. Baskar, B. De Schutter, and H. Hellendoorn
}

If you want to cite this report, please use the following reference instead:

\begin{abstract}
L.D. Baskar, B. De Schutter, and H. Hellendoorn, "Model-based predictive traffic control for intelligent vehicles: Dynamic speed limits and dynamic lane allocation," Proceedings of the 2008 IEEE Intelligent Vehicles Symposium (IV'08), Eindhoven, The Netherlands, pp. 174-179, June 2008.
\end{abstract}




\title{
Model-Based Predictive Traffic Control for Intelligent Vehicles: Dynamic Speed Limits and Dynamic Lane Allocation
}

\author{
Lakshmi Dhevi Baskar, Bart De Schutter, and Hans Hellendoorn
}

\begin{abstract}
We consider traffic management and control approaches for automated highway systems with a combination of intelligent vehicles and roadside controllers. The vehicles are organized in platoons with short intraplatoon distances, and larger distances between platoons. Moreover, all vehicles are assumed to be automated, i.e., throttle, braking, and steering commands are determined by an automated on-board controller. Within a platoon the vehicles coordinate their actions so as to maintain a small but safe intervehicle distance, using adaptive cruise control methods. Platoon leaders receive speed set-points and lane change commands from the roadside controller. We propose a model-based predictive control (MPC) approach to determine appropriate speed limits and lane allocations for the platoons. In general, this results in mixed-integer optimization problems. We discuss some methods to solve these problems suboptimally on-line. The proposed approach is then applied to a simple simulation example in which the aim is to minimize the total time all vehicles spend in the network by optimally assigning dynamic speed limits and lane changes.
\end{abstract}

\section{INTRODUCTION}

Due to the ever-increasing demand for mobility and transportation, traffic congestion is a growing problem throughout the world. There are many possible ways to reduce the frequency and impact of traffic jams (such as building new roads, introducing road pricing policies, stimulating modal shift, promoting public transportation, and so on). On the short term one of the most promising approaches is the use of advanced traffic management and control methods in which control measures such as traffic signals, dynamic route information panels, ramp metering installations, dynamic speed limits, etc. are used to control the traffic flows and to prevent or to reduce traffic jams, or more generally to improve the performance of the traffic system. Possible performance measures in this context are throughput, travel times, safety, fuel consumption, robustness, etc.

The currently implemented traffic management and control approaches primarily make use of roadside and infrastructure-based equipment (including sensors, actuators, and traffic control centers). However, due to advances in the field of telecommunication, control theory, and information systems, and the increasing implementation of onboard sensing, actuating, and telecommunication devices, next-generation traffic management concepts that incorporate intelligence in both roadways and vehicles will become feasible, such as Intelligent Transportation Systems (ITS)

L.D. Baskar, B. De Schutter, and H. Hellendoorn are with the Delft Center for Systems and Control, Delft University of Technology, Delft, The Netherlands. B. De Schutter is also with the Marine \& Transport Technology department of Delft University of Technology. \{l.d.baskar, j.hellendoorn\}@tudelft.nl, b@deschutter.info
[1], Intelligent Vehicle Highway Systems [2], Automated Highway Systems (AHS) [3], or Cooperative Vehicle Infrastructure Systems (CVIS). In the reminder of this paper we will use IVHS as a generic word for indicating (a mixture of) ITS, IVHS, AHS, and CVIS.

For better coordination of traffic network activities, IVHS also distribute the intelligence between the vehicles and the roadside infrastructure. Moreover, in IVHS the driver tasks are shifted away from driver to the vehicle since autonomous control of driving tasks can substantially improve the traffic flow [4]. Indeed, complete automation allows to arrange the vehicles in closely spaced groups called "platoons". In a platoon, the first vehicle is called "leader" and the remaining are called "followers". In the platooning approach cars travel on the highway in platoons with small distances (e.g., $2 \mathrm{~m}$ ) between vehicles within the platoon, and much larger distances (e.g., 30-60 m) between different platoons. High speeds and short intraplatoon spacings allow more vehicles to be accommodated on the network, which substantially increases the maximal traffic flows. Due to the very short intraplatoon distance this approach requires automated distance keeping since human drivers cannot react fast enough to guarantee adequate safety. So in IVHS every vehicle contains an automatic system that can take over the driver's responsibilities in steering, braking, and throttle control.

In this paper, we deal traffic congestion problems by using a variant of IVHS in which the monitoring and control handles offered by automated intelligent vehicles are combined with those of the roadside infrastructure. More specifically, we integrate the platooning approach with conventional traffic control measures such as dynamic speed limits, route guidance, ramp metering, etc. The overall control framework we use is the hierarchical framework we have presented in [5] and which is closely related to the PATH framework [6]. We will in particular focus on the control layer that manages the different platoons in the traffic network and that controls the speeds and lane changes of the platoons.

This paper is organized as follows. Section II starts with a short survey on intelligent vehicles (IVs) and IV-based traffic management. We also recapitulate the hierarchical IV-based traffic control framework adopted in this paper. Section III describes the model-based predictive control design method that will be used to determine optimal speed limits and lane change commands for the platoons. In Section IV we apply the proposed approach to a case study based on simulations and we highlight the potential effects of the proposed approach on the traffic flow performance. Conclusions and topics for future research are discussed in Section V. 


\section{INTELLIGENT VEHICLES AND IV-BASED TRAFFIC MANAGEMENT}

\section{A. Intelligent vehicles and IV-based traffic control measures}

Intelligent Vehicles (IVs) are equipped with control systems that can sense the environment around the vehicle and that result in a more efficient vehicle operation by assisting the driver or by taking complete control of the vehicle [7]. Depending on the level of support provided to the driver, we can divide IV application areas into three categories:

- advisory systems (which use optic or acoustic systems to provide an advisory or a warning to the drivers),

- semi-autonomous systems (which use haptic measures to assist the driver or to take partial control of the vehicle),

- fully autonomous systems (which take complete control of vehicle operation).

Note that the platoon-based approach used in this paper assumes that all IVs are fully autonomous.

We will now briefly discuss the main IV technologies that support and improve the platooning concept by allowing vehicle-vehicle and vehicle-roadside coordination [7], [8]:

1) Intelligent Speed Adaptation (ISA): ISA is based on a speed limiter incorporated within each car and which can take into account speed limit restrictions, can adjust the maximum driving speed to the speed limit specified by the roadside infrastructure, and can provide feedback to the driver or take autonomous action when that speed limit is exceeded. ISA systems could use fixed or dynamic speed limits. In the fixed case, the driver is informed about the speed limit, which could be obtained from a static database. Dynamic speed limits take into account the current road conditions such as bad weather, slippery roads, or major incidents before prescribing the speed limit.

2) Adaptive Cruise Control (ACC): An ACC system is a radar-based system that extends the conventional cruise control and that is designed to sense the immediate vehicle in front on the lane, and to automatically adjust the speed of the equipped vehicle to match the speed of the preceding vehicle and to maintain a safe intervehicle distance [9]. If there is no vehicle in front, then ACC controller retains a preset free-flow speed that can be selected by driver.

Cooperative ACC is a further enhancement of ACC systems that uses existing communication technologies (e.g., wireless technologies) to obtain real-time information about the speed, acceleration, etc. of the preceding vehicle. Cooperative ACC equipped vehicles can exchange the information much quicker and allow to set the safe minimum headway as small as $0.5 \mathrm{~s}$. With this reduced headways between vehicles the maximal traffic flow can be augmented even further.

3) Dynamic route planning and guidance: A route guidance system advises a driver about the "best" route he can take to reach his requested destination [10]. Recommended routes may be calculated within the equipped vehicle ("route planning") or communicated to the vehicle from the local traffic center ("route guidance"). When the possible routes are computed based on the average conditions of the traffic

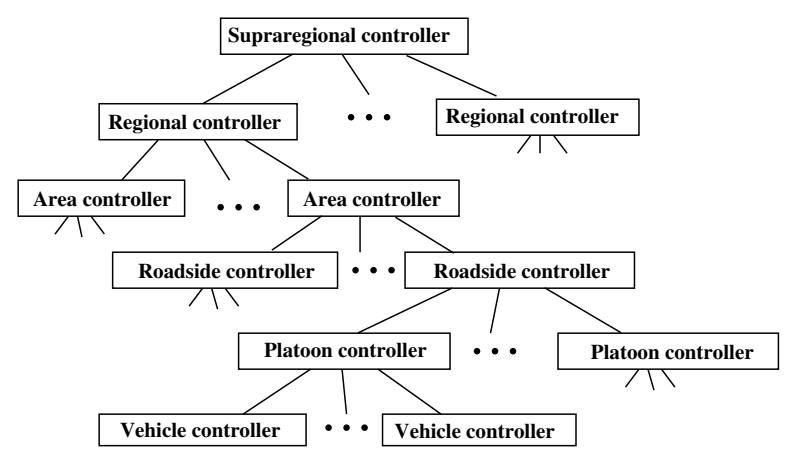

Fig. 1. IV-based framework.

network, this scheme is referred to as static route guidance. If the existing traffic conditions and frequent updates of travel times based on real-time traffic are taken into account when computing the route recommendations, then this is called a dynamic route planning system.

The IV-based approach we propose includes ISA with dynamic speed limits, cooperative ACC, and dynamic route guidance, as well as conventional infrastructure-based traffic control measures.

\section{B. Hierarchical framework for IV-based traffic management}

In this section, we will briefly present the hierarchical control framework we have proposed in [5]. This framework distributes the intelligence between roadside infrastructure and vehicles, assigns the traffic control actions based on platoons rather than on highway sections (as is done in PATH), and uses the IV-based control measures mentioned in Section II to improve the traffic flow.

The proposed control architecture is based on the platoon concept and consists of a multi-level control structure with local controllers at the lowest level and one or more higher supervisory control levels. A general structure of proposed architecture is a distributed hierarchy as shown in Figure 1.

The layers of the framework can be characterized as follows:

1) Higher-level controllers: The higher-level controllers (such as area, regional, and supraregional controllers) provide network-wide coordination of the lower-level and middlelevel controllers. For instance, the activities of a group of roadside controllers could be supervised by an area controller. In turn, a group of area controllers could be supervised or controlled by regional controllers, and so on.

2) Roadside controllers: The roadside controllers use IV-based control measures to improve the traffic flow. A roadside controller may control a part of a highway, an entire highway, or a collection of highways. Each platoon in the highway network appears as a one single entity to the roadside controller and therefore can be managed more efficiently. The main tasks of the roadside controllers are to assign desired speeds for each platoon, safe distances to avoid collisions between platoons, desired platoon sizes depending on the traffic conditions, to provide dynamic route guidance for the platoons, metering values on the on-ramps 
and off-ramps (ramp metering), and also to instruct for merges, splits, and lane changes of platoons.

3) Platoon controllers: The platoon controllers receive commands from the roadside controllers and are responsible for control and coordination of each intelligent vehicle inside the platoon. The platoon controller is mainly concerned with actually executing the interplatoon maneuvers (such as merges with other platoons, splits, and lane changes) and intraplatoon activities (such as maintaining safe intervehicle distances).

4) Vehicle controllers: The vehicle controllers present in each vehicle receive commands from the platoon controllers (e.g., set-points or reference trajectories for speeds (ISA), headways (ACC), and paths) and they translate these commands into control signals for the vehicle actuators such as throttle, braking, and steering actions.

The main improvements and extensions of the new framework when compared to existing frameworks are [5]:

- It enables the integration of in-vehicle IV-based traffic control measures (such as ISA and ACC) and roadside traffic control measures (such as ramp metering, variable speed limits, dynamic route guidance, etc.).

- Platoon sizes are variable and optimal values can be determined by the roadside controllers.

- The framework can be integrated with a model-based predictive control strategy that determines optimal traffic control measures in a receding horizon approach and that will be described in the next section.

- The hierarchical structure enhances the scalability and allows to manage and to control traffic flows in largescale networks.

\section{Model Predictive Control for Intelligent VEHICLES}

The actual control strategy to be used in our approach is a model-based predictive control approach called Model Predictive Control (MPC),

\section{A. Model Predictive Control - General framework}

Model Predictive Control (MPC) [11], [12] has originated in the process industry and it has already been successfully implemented for many industrial applications.

We will now briefly summarize the main ideas behind MPC (see Figure 2). In Section III-B they will then be made more concrete for MPC-based traffic control using intelligent vehicles and platoons in combination with the IV-based traffic control measures of Section II-A and the hierarchical framework of Section II-B.

MPC is based on (on-line) optimization and uses an explicit prediction model to obtain the optimal actions for the control measures. Let $T_{\mathrm{c}}$ be the control sampling interval, i.e., the (constant) time interval between two updates of the control signal settings. At each time step $k$ (corresponding to the time instant $t=k T_{\mathrm{c}}$ ), the controller measures or determines the current state $x(k)$ of the system and uses a model of the system to predict the behavior of the system over an interval $\left[k, k+N_{\mathrm{p}}\right]$, where $N_{\mathrm{p}}$ is called the prediction horizon, as

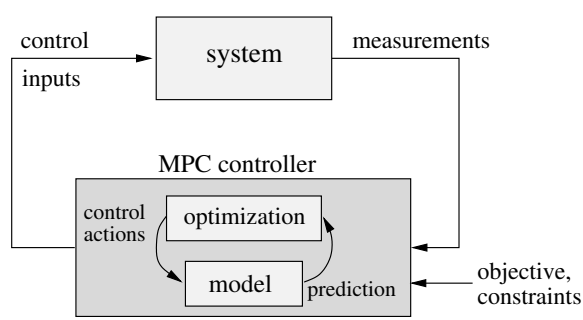

Fig. 2. Schematic representation of MPC.

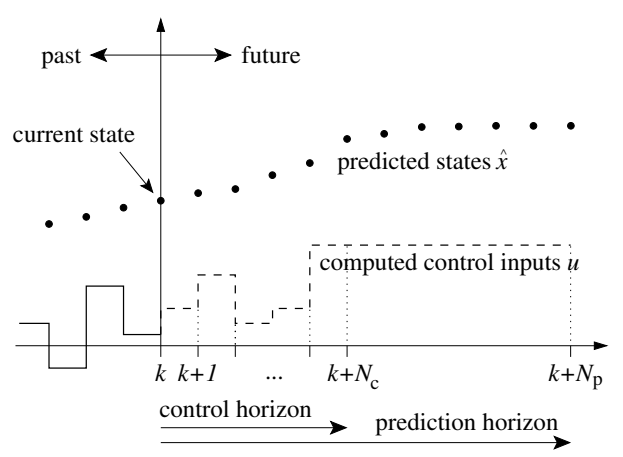

Fig. 3. Representation of the MPC control scheme.

shown in Figure 3. Next, the controller solves an open-loop optimal control problem to determine the optimal control inputs $u(k), \ldots, u\left(k+N_{\mathrm{p}}-1\right)$ that minimize a performance criterion $J(k)$ over the prediction period $\left[k, k+N_{\mathrm{p}}\right]$, subject to the operational constraints. To reduce the computational complexity of the problem, one often introduces a constraint of the form $u(k+j)=u(k+j-1)$ for $j=N_{\mathrm{c}}, \ldots, N_{\mathrm{p}}-1$, where $N_{\mathrm{c}}\left(<N_{\mathrm{p}}\right)$ is called the control horizon. When the optimal values are found by the controller, the control actions are applied in a receding horizon fashion. This is done by applying only the first control sample $u(k)$ of the optimal control sequence to the system. Next, the prediction horizon is shifted one step forward and the prediction and optimization procedure over the shifted horizon are repeated using new system measurements.

The receding horizon approach introduces a feedback mechanism, which allows to reduce the effects of possible disturbances and mismatch errors between the actual realworld traffic flows and the predicted traffic flows.

\section{B. IV-based MPC}

There are several reasons why MPC is probably the most applied advanced control technique in the process industry:

- MPC can easily handle multi-input multi-output processes, processes with large time-delays, and unstable processes.

- It is an easy-to-tune method.

- MPC can handle constraints on the inputs and the outputs of the process in a systematic way during the design and the implementation of the controller.

- MPC can handle changes in system parameters or system structure (including sensor or actuator failures) 
by regularly updating the parameters and the structure of the prediction model.

However, the use of MPC is not limited to the process industry. The many advantages that MPC offers are also relevant for traffic control. In fact, MPC has already been extended to conventional roadside-based non-IV traffic management [13], [14]. However, MPC can also be used for traffic management and control with intelligent vehicles. In particular, MPCbased traffic control offers several advantages on top of the ones mentioned above:

- It allows a network-wide coordination of the various traffic control measures.

- Using upstream and downstream measurements, historical data, and information gathered from the intelligent vehicles, we can anticipate on future changes in traffic demands, weather conditions, etc.

In the context of the hierarchical IV-based traffic control framework of Figure 1 MPC can be applied at almost all levels (except maybe the vehicle control level where the online optimization requirements of MPC might interfere with the short control sample times at this level). In this paper, we will in particular focus on the roadside controller level. Typical control sampling times at this level range from $10 \mathrm{~s}$ to $1 \mathrm{~min}$.

There exists a wide range of traffic models [15], [16]. An important factor that determines the choice of the prediction model to be used in MPC is the trade-off between accuracy of the model and the computational complexity since at each time step $k$ the model will be simulated repeatedly within the on-line optimization algorithm. As a consequence, very detailed microscopic traffic simulation models are usually not suited as MPC prediction model. Instead, usually more aggregate macroscopic models are applied. For instance, the roadside controller could use a model in which the platoons are the basic simulation entities instead of individual cars. Also note that MPC is a modular approach so that in case a given prediction model does not perform well, it can easily be replaced by another prediction model.

Possible performance criteria $J(k)$ at the roadside controller level are minimizing the total time spent in a traffic network, total throughput, total fuel consumption, safety or a combination of these. In this paper we will in particular consider the total time spent by all the vehicles in the network:

$$
J_{\mathrm{TTS}}(k)=\sum_{j=0}^{N_{\mathrm{p}}} n_{\mathrm{veh}}(k+j) T_{\mathrm{c}},
$$

where $n_{\mathrm{veh}}(k+j)$ is the number of vehicles that are present in the network at time $t=(k+j) T_{\mathrm{c}}$. Moreover, in order to prevent oscillations and frequent shifting in the control signals, there is also often a penalty on variations in the control signal $u$, which results in the penalty term

$$
J_{\mathrm{pen}}(k)=\sum_{j=0}^{N_{\mathrm{p}}}\|u(k+j)-u(k+j-1)\|_{Q}
$$

that has to be added to the performance criterion $J(k)$, where $Q$ is a positive semi-definite matrix and $\|v\|_{Q}=v^{\mathrm{T}} Q v$ for a vector $v$. This leads to the total MPC objective function

$$
J_{\text {tot }}(k)=J(k)+J_{\text {pen }}(k)
$$

at time step $k$.

Possible control measures that can be determined by the roadside controller are then dynamic speeds limits for the platoons (ISA), lane changes ${ }^{1}$, platoons sizes, merge and split operations, as well as settings for the roadside-based measures such as ramp metering rates, lane closures, shoulder lane openings (on highways) and traffic signal settings (in urban environments).

Typical constraints for the roadside controller are then minimum separation between vehicles and platoons, maximum platoons lengths, minimum and maximum speeds, minimum headways, minimum and maximum metering rates and green or red times, etc.

\section{Optimization methods}

Computing the optimal control action (i.e., solving the MPC optimization problem) is the most demanding operation in the MPC approach. For our framework, the MPC approach could give rise to discrete (i.e., integer), continuous, or mixed-integer optimization problems depending on the nature of the control measures as we could have both continuous variables (dynamic speed limits, ramp metering rates, green times, etc.) and discrete variables (platoon size, lane allocations, lane closures, etc.). The resulting optimization problems have to be solved on-line. Hence, a proper choice of optimization techniques that would suit the nature of the problem has to be made.

In general, the optimization problems resulting from IVbased control will be nonlinear and nonconvex, which implies that global or multi-start local optimization methods are required. Continuous optimization problems can be solved using multi-start sequential quadratic programming [17] or pattern search [18]. Integer and mixed-integer optimization problems could be solved using genetic algorithms [19], simulated annealing [20], or branch-and-bound methods [21].

\section{CASE STUdy}

The effectiveness of any traffic control strategy can be evaluated by verifying whether the controller is able to resolve or to eliminate the possible traffic problems in a better way than other approaches. Some of the problems that could degrade the traffic performance are congestion, capacity drop, or blocking scenario. In this section, we present a simple case study in which the MPC control strategy described in Section III is used by the roadside controller layer. First, we will describe the scenario used to evaluate the performance of the proposed approach, the models used by MPC, and other implementation details.

\footnotetext{
${ }^{1}$ The routes of the platoons would then determined by both the area controllers (as regards which route to follow within the area under control) and by the roadside controller (as regards which route to follow within the highway segments under the control of the given roadside controller)
} 


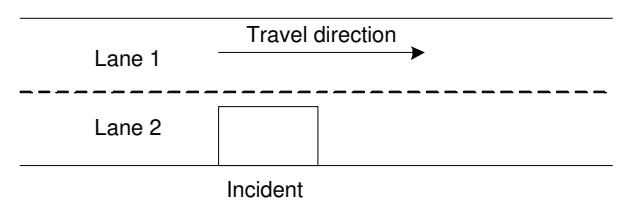

Fig. 4. Set-up of the case study.

Next, we will discuss and analyze the results obtained from the simulations for the considered scenario.

\section{A. Set-up}

We use a basic set-up as a test-bed for implementing our proposed framework consisting of a $10 \mathrm{~km}$ highway stretch with two lanes and without any on-ramp and off-ramp (see Figure 4). We will compare three different situations:

- uncontrolled traffic (with human drivers),

- controlled traffic with human drivers and autonomous ISA as control measure ${ }^{2}$,

- IV-based traffic with platoons.

For the sake of simplicity all vehicles are assumed to be of same length $(4 \mathrm{~m})$. In the IV-based case we assume that all the vehicles are fully automated IVs equipped with advanced communication and detection technologies such as in-vehicle computers and sensors and with on-board ACC and ISA controllers. Both human driven vehicles and platoons are allowed to change lanes, provided suitable gaps are found in the targeted lane.

\section{B. Scenario}

In general, traffic congestion occurs when the available network resources are not sufficient to handle the traffic demand, or due to irregular occurrences, such as traffic incidents. In practice, traffic jams or congestion result in capacity drop. A capacity drop is defined as a phenomenon that causes the expected maximum outflow from the jammed traffic to be less than in the case of free-flow traffic. This is mainly caused by the delay in reaction time and increased intervehicle distance (time headway) when vehicles start to exit from a traffic jam. For human drivers the capacity drop is typically of the order of $2-5 \%$. With fully automated vehicles the capacity drop can be reduced to almost $0 \%$.

We simulate a period of $30 \mathrm{~min}$ starting at time $t_{\text {start }}=$ $7 \mathrm{~h} 30 \mathrm{~min}$ and ending at time $t_{\text {end }}=8 \mathrm{~h} 00 \mathrm{~min}$. In the proposed scenario an incident occurs at position $x=500 \mathrm{~km}$ in lane 2, and exists for an interval of $15 \mathrm{~min}$, starting at time $t_{1}=t_{\text {start }}=7 \mathrm{~h} 30 \mathrm{~min}$ and ending at time $t_{2}=7 \mathrm{~h} 45 \mathrm{~min}$. During this interval, the maximum outflow from the incident position $x$ is less when compared to free-flow traffic due to the capacity drop. The value of this capacity drop in our case is around $2 \%$. After time $t_{2}$, the traffic flow at position $x$ returns slowly to the regular level.

\footnotetext{
${ }^{2}$ So we assume that ISA limits the speed in a hard way and that human drivers cannot surpass the imposed speed limit. Note that with advisory ISA the performance can be expected to be somewhat worse depending on the compliance level of the drivers (advisory ISA typically result in a relative variance of about $5 \%$ of the speeds around the ISA speed limit [22]).
}

\section{Models}

As indicated above we are interested in comparing the simulation results obtained for the same scenario using human driving (both without and with control) and using our platoon-based hierarchical approach. For this purpose, we have developed simulation models in Matlab for human driving and platoon driving. For the sake of simplicity and to avoid calibration, we have used the same model for both simulation and prediction purposes in this simulation study.

The human driver behavior is captured using a standard car-following model [23] and for platoon driving model, we use a PID controller as ACC controller to maintain a safe distance for the vehicles inside the platoon and to track the speed of the platoon leader (which in its turn is influenced by the dynamic speed limit provided by the roadside controller).

The time step for the simulations is set at $1 \mathrm{~s}$.

\section{Control problem}

The goal of our traffic controller is to improve the traffic performance. The objective that we consider is minimization of the total time spent by all the vehicles in the network (see (1)) and we use dynamic speed limits and lane changes as the control handles. So the control signal $u$ for the MPC problem of time step $k$ includes speed limits for all platoons that are in the network at time $k$ as well as their lane allocations. We have also included a penalty term $J_{\text {pen }}(k)$ of the form (2) with $Q_{i i}=2$ if $u_{i}$ is a lane change control input and $Q_{i i}=0.02$ if $u_{i}$ is a speed control input. We consider a maximum speed of $120 \mathrm{~km} / \mathrm{h}$ for both the human drivers and the platoon leaders. As we focus on dynamic speed limits and lane changes as the control handles, the platoon size is not considered to be a control variable, but it is kept fixed at 5 for all platoons.

The control sampling time $T_{\mathrm{c}}$ is set at $1 \mathrm{~min}$. For the prediction horizon $N_{\mathrm{p}}$ we have taken a value that corresponds to $20 \mathrm{~min}$, and for the control horizon $N_{\mathrm{c}}$ we have selected a value that corresponds to $2 \mathrm{~min}$ so as to limit the number of optimization variables.

For the optimization we have used the fmincon command of the Matlab Optimization toolbox for the continuous optimization problems (i.e., the determination of the speeds for the controlled human case) and the glcFast command of the Matlab/Tomlab toolbox for the mixed-integer optimization problems. The fmincon command implements Sequential Quadratic Programming and the glcFast command implements an extended version of the DIRECT algorithm of [24]. Both methods have been executed multiple times so as to get a good approximation of the optimal solution.

\section{E. Results and analysis}

For the given scenario with an incident, the results obtained from human driving with ISA control and without control, and the hierarchical traffic controller for IV-based platoons are reported in Table I. In particular, we report the Total Time Spent (TTS) by all vehicles in the network during 


\begin{tabular}{|l|c|c|}
\hline Case & TTS (veh.min) & Relative improvement \\
\hline uncontrolled case & 1465 & $0 \%$ \\
controlled (human drivers) & 1367 & $6.69 \%$ \\
controlled (platoons) & 1320 & $9.90 \%$ \\
\hline
\end{tabular}

TABLE I

RESULTS OF THE THREE APPROACHES. THE RELATIVE IMPROVEMENT IS COMPUTED WITH RESPECT TO THE UNCONTROLLED CASE.

the entire simulation period:

$$
J_{\mathrm{TTS}}(k)=\sum_{k=0}^{N_{\mathrm{sim}}} n_{\mathrm{veh}}(k) T_{\mathrm{c}},
$$

where $N_{\text {sim }}$ represents the simulation length (expressed in number of control steps), so the total simulation time is $N_{\text {sim }} T_{\mathrm{c}}$, and hence $N_{\text {sim }}=30$.

Clearly, the IV-based traffic with platoons results in the best performance with an improvement of about $10 \%$ with respect to the uncontrolled case. The results can be explained as follows.

In the uncontrolled case with human drivers, when there are no vehicles in front of the driver or if there is enough space between two drivers, then the drivers maintain their desired speed. But when the driver is confronted with an incident, then he can either decelerate in order to avoid collision with the incident or if there exists a desired gap in the next lane, then he can change lane to avoid waiting.

For the same scenario but with the human driver and ISA control, our approach can predict the presence of the incident and prevent it or diminish the negative impacts by slowing down the vehicles before they reach the incident. In addition, in the IV-based control approach with platoon, our approach also allocates appropriate lane for each platoon to avoid confronting the incident.

\section{CONClusions And Future Work}

We have presented how a model predictive control approach can be used to determine speed and lane change commands for platoons of intelligent vehicles (IVs) by roadside controllers within a hierarchical framework. The proposed approach has been illustrated using a case study based on simulations. The results of the case study highlight the potential benefits and improvements that can be obtained by using an IV-based traffic control approach with interaction and integration between the roadside infrastructure and the fully automated IVs.

Future research topics include: additional and more extensive case studies, inclusion of additional control measures apart from speed limits and lane changes, taking model uncertainty, disturbances, and inaccurate predictions about future demands into account, explicit consideration of the other levels in the control hierarchy, and extension of the approach to larger networks.

\section{Acknowledgments}

Research supported by the VIDI project "Multi-Agent Control of Large-Scale Hybrid Systems" (DWV.6188) of the Dutch Technology Foundation STW, Applied Science division of NWO and the Technology Programme of the Dutch Ministry of Economic Affairs, the BSIK projects "Transition to Sustainable Mobility (TRANSUMO)" and "Next Generation Infrastructures (NGI)", the Transport Research Centre Delft, and the Delft Research Center Next Generation Infrastructures.

\section{REFERENCES}

[1] J. M. Sussman, "Intelligent vehicle highway systems:challenge for the future," IEEE Micro, vol. 1, no. 14-18, pp. 101-104, Jun. 1993.

[2] P. Varaiya and S. E. Shladover, "Sketch of an IVHS systems architecture," in Proceedings of the IEEE Vehicle Navigation and Information Systems, Dearborn, MI, Oct. 1991, pp. 909-922.

[3] R. Horowitz and P. Varaiya, "Control design of an automated highway system," Proceedings of the IEEE : Special Issue on Hybrid Systems, vol. 88, no. 7, pp. 913-925, Jul. 2000.

[4] P. Varaiya, "Smart cars on smart roads" IEEE Transactions on Automatic Control, vol. 38, no. 2, pp. 195-207, Feb. 1993.

[5] L. Baskar, B. De Schutter, and H. Hellendoorn, "Hierarchical traffic control and management with intelligent vehicles," in Proceedings of the 2007 IEEE Intelligent Vehicles Symposium (IV'07), Istanbul, Turkey, Jun. 2007, pp. 834-839.

[6] S. Shladover, C. A. Desoer, J. K. Hedrick, M. Tomizuka, J. Walrand, W. B. Zhang, D. H. McMahon, H. Peng, S. Sheikholeslam, and N. McKeown, "Automatic vehicle control developments in the PATH program," IEEE Transactions on Vehicle Technology, vol. 40, no. 1, pp. 114-130, Feb. 1991.

[7] R. Bishop, Intelligent Vehicles Technology and Trends. Artech House, 2005.

[8] L. S. Comte, "New systems: new behaviour?" Transportation Research Part $F$, vol. 3, no. 2, pp. 95-111, May 2000.

[9] L. C. Davis, "Effect of adaptive cruise control systems on traffic flow," Physical Review E, vol. 69, pp. 1-8, 2004.

[10] D. Watling and T. van Vuren, "The modelling of dynamic route guidance systems," Transportation Research Part C, vol. 1, no. 2, pp. 159-182, Jun. 1993.

[11] J. M. Maciejowski, Predictive Control with Constraints. Harlow, England: Prentice Hall, 2002.

[12] E. F. Camacho and C. Bordons, Model Predictive Control in the Process Industry. Berlin, Germany: Springer-Verlag, 1995.

[13] A. Hegyi, B. De Schutter, and J. Hellendoorn, "Optimal coordination of variable speed limits to suppress shock waves," IEEE Transactions on Intelligent Transportation Systems, vol. 6, no. 1, pp. 102-112, Mar. 2005.

[14] T. Bellemans, B. De Schutter, and B. De Moor, "Model predictive control for ramp metering of motorway traffic: A case study," Control Engineering Practice, vol. 14, no. 7, pp. 757-767, Jul. 2006.

[15] C. Daganzo, Fundamentals of Transportation and Traffic Operations. Pergamon Press, 1997.

[16] S. P. Hoogendoorn and P. H. L. Bovy, "State-of-the-art of vehicular traffic flow modelling," Proceedings of the Institution of Mechanical Engineers, Part I: Journal of Systems and Control Engineering, vol. 215, no. 4, pp. 283-303, Aug. 2001.

[17] P. M. Pardalos and M. G. C. Resende, Handbook of Applied Optimization. Oxford, UK: Oxford University Press, 2002.

[18] C. Audet and J. E. Dennis Jr., "Analysis of generalized pattern searches," SIAM Journal on Optimization, vol. 13, no. 3, pp. 889903, 2007.

[19] L. Davis, Handbook of Genetic Algorithms. New York: Van Nostrand Reinhold, 1991.

[20] R. Eglese, "Simulated annealing: A tool for operations research," European Journal of Operational Research, vol. 46, pp. 271-281, 1990.

[21] C. Cordier, H. Marchand, R. Laundy, and L. Wolsey, "bc-opt: A branch-and-cut code for mixed integer programs," Mathematical Programming, Series A, vol. 86, no. 2, pp. 335-353, 1999.

[22] M. Hjälmdahl, S. Almqvist, , and A. Várhelyi, "Speed regulation by in-car active accelerator pedal - Effects on speed and speed distribution," IATSS Research, vol. 26, no. 2, pp. 60-66, May 2002.

[23] D. Gazis, R. Herman, and B. Potts, "Car-following theory of steady traffic flow," Operations Research, vol. 7, no. 2, pp. 499-505, Jun. 1959.

[24] D. R. Jones, "DIRECT," in Encyclopedia of Optimization. Kluwer Academic Publishers, 2001 